\title{
A time domain characterization of vector-valued subspace weak Gabor bi-frames
}

Jing Zhao ${ }^{1}$ and Yun-Zhang Li ${ }^{1^{*}}$

"Correspondence:

yzlee@bjut.edu.cn

${ }^{1}$ College of Applied Sciences,

Beijing University of Technology,

Beijing, P.R. China

\section{每 Springer}

\begin{abstract}
The construction of bi-frames is a fundamental problem in frame theory. Due to their wide applications, the study of vector-valued frames and subspace frames has interested many mathematicians in recent years. In this paper, we introduce the weak Gabor bi-frame (WGBF) in vector-valued subspaces, characterize WGBFs on the time domain, and present some examples.
\end{abstract}

MSC: Primary 42C40; secondary 42C15

Keywords: Weak Gabor bi-frame; Vector-valued frame; Vector-valued subspace frame

\section{Introduction}

The concept of frame was introduced by Duffin and Schaeffer in [1], which dealt with nonharmonic Fourier series, and Gabor frames date back to [2]. Nowadays Gabor frames have been widely applied in signal processing [3-6]. They have been studied quite extensively on the whole space $L^{2}(\mathbb{R})$. Vector-valued frames were also called superframes, they were introduced in [7] by Han and in [8,9] by Balan in the context of signal multiplexing, which means encoding several signals as a single one with the purpose of sharing a communication channel. In some structured cases with orthogonal windows known as superframes it is possible to find a Nyquist rate defining a phase transition between super-Riesz sequences and superframes. This was done in [10,11] for the Gabor case, and in [12] for the wavelet case, following the constructions of vector-valued wavelet transforms [12-14]. In applications, when signals belong to a subspace of $L^{2}\left(\mathbb{R}, \mathbb{C}^{L}\right)$, one would like to perform a Gabor analysis of the signals in the most efficient way, while still preserving all the features of the observed data. That is why subspace Gabor analysis has interested many mathematicians. The literature $[10,11,15-34]$ has considered Gabor frames for $L^{2}\left(\mathbb{R}, \mathbb{C}^{L}\right)$ and their subspaces.

We denote by $\mathbb{Z}$ the set of integers, and by $\mathbb{N}$ the set of positive integers. Let $\mathcal{H}$ be a separable Hilbert space, and $\left\{e_{i}\right\}_{i \in \mathcal{I}}$ be an at most countable sequence in $\mathcal{H}$. The sequence $\left\{e_{i}\right\}_{i \in \mathcal{I}}$ is called a frame (tight frame; Parseval frame) for $\mathcal{H}$ if there exist positive constants

(c) The Author(s) 2018. This article is distributed under the terms of the Creative Commons Attribution 4.0 International License (http://creativecommons.org/licenses/by/4.0/), which permits unrestricted use, distribution, and reproduction in any medium, provided you give appropriate credit to the original author(s) and the source, provide a link to the Creative Commons license, and indicate if changes were made. 
$A$ and $B$ such that

$$
A\|f\|^{2} \leq \sum_{i \in \mathcal{I}}\left|\left\langle f, e_{i}\right\rangle\right|^{2} \leq B\|f\|^{2}
$$

for $f \in \mathcal{H}(A=B ; A=B=1)$; and it is called a Bessel sequence in $\mathcal{H}$ if the right-hand side inequality holds. Let $\left\{e_{i}\right\}_{i \in \mathcal{I}}$ be a frame for $\mathcal{H}$. A frame $\left\{\tilde{e}_{i}\right\}_{i \in \mathcal{I}}$ for $\mathcal{H}$ is called a dual of $\left\{e_{i}\right\}_{i \in \mathcal{I}}$ if

$$
f=\sum_{i \in \mathcal{I}}\left\langle f, \tilde{e}_{i}\right\rangle e_{i} \quad \text { for } f \in \mathcal{H}
$$

By a simple argument, we see that (1) is equivalent to

$$
f=\sum_{i \in \mathcal{I}}\left\langle f, e_{i}\right) \tilde{e}_{i}
$$

So, in this case, we also say $\left(\left\{e_{i}\right\}_{i \in \mathcal{I}},\left\{\tilde{e}_{i}\right\}_{i \in \mathcal{I}}\right)$ is a pair of dual frames (bi-frame) for $\mathcal{H}$. And by a standard argument, $\left(\left\{e_{i}\right\}_{i \in \mathcal{I}},\left\{\tilde{e}_{i}\right\}_{i \in \mathcal{I}}\right)$ is a bi-frame for $\mathcal{H}$ if and only if $\left\{e_{i}\right\}_{i \in \mathcal{I}}$ and $\left\{\tilde{e}_{i}\right\}_{i \in \mathcal{I}}$ are Bessel sequences, and

$$
\langle f, g\rangle=\sum_{i \in \mathcal{I}}\left\langle f, \tilde{e}_{i}\right\rangle\left\langle e_{i}, g\right\rangle \quad \text { for } f, g \in \mathcal{H}
$$

Given $L \in \mathbb{N}$ and $a, b>0$, let $L^{2}\left(\mathbb{R}, \mathbb{C}^{L}\right)$ be the Hilbert space of vector-valued square integrable functions endowed with the inner product

$$
\langle\mathbf{f}, \tilde{\mathbf{f}}\rangle=\sum_{l=1}^{L} \int_{\mathbb{R}} f_{l}(t) \overline{\tilde{f}_{l}(t)} d t
$$

for $\mathbf{f}=\left(f_{1}, f_{2}, \ldots, f_{L}\right), \tilde{\mathbf{f}}=\left(\tilde{f}_{1}, \tilde{f}_{2}, \ldots, \tilde{f}_{L}\right) \in L^{2}\left(\mathbb{R}, \mathbb{C}^{L}\right)$. The modulation operator $E_{m b}$ and translation operator $T_{n a}$ on $L^{2}\left(\mathbb{R}, \mathbb{C}^{L}\right)$ with $m, n \in \mathbb{Z}$ are defined by

$$
E_{m b} \mathbf{f}=\left(E_{m b} f_{1}, E_{m b} f_{2}, \ldots, E_{m b} f_{L}\right) \quad \text { and } \quad T_{n a} \mathbf{f}=\left(T_{n a} f_{1}, T_{n a} f_{2}, \ldots, T_{n a} f_{L}\right)
$$

for $\mathbf{f} \in L^{2}\left(\mathbb{R}, \mathbb{C}^{L}\right)$, respectively, where

$$
E_{m b} f(\cdot)=e^{2 \pi i m b} f(\cdot) \quad \text { and } \quad T_{n a} f(\cdot)=f(\cdot-n a)
$$

for $f \in L^{2}(\mathbb{R})$. Obviously, $L^{2}\left(\mathbb{R}, \mathbb{C}^{L}\right)$ is the $L$-fold direct sum of $L^{2}(\mathbb{R})$. Throughout this paper, $f_{l}$ denotes the $l$ th component of $\mathbf{f} \in L^{2}\left(\mathbb{R}, \mathbb{C}^{L}\right)$, and $\mathcal{G}(\mathbf{g}, a, b)$ denotes the Gabor system:

$$
\mathcal{G}(\mathbf{g}, a, b)=\left\{E_{m b} T_{n a} \mathbf{g}: m, n \in \mathbb{Z}\right\}
$$

for $\mathbf{g} \in L^{2}\left(\mathbb{R}, \mathbb{C}^{L}\right)$. A set $S$ in $\mathbb{R}$ with positive measure is said to be $a \mathbb{Z}$-periodic if $S+$ an $=S$ for $n \in \mathbb{Z}$. For such $S$, we denote by $L^{2}\left(S, \mathbb{C}^{L}\right)$ the closed subspace of $L^{2}\left(\mathbb{R}, \mathbb{C}^{L}\right)$ of the form

$$
L^{2}\left(S, \mathbb{C}^{L}\right)=\left\{\mathbf{f} \in L^{2}\left(\mathbb{R}, \mathbb{C}^{L}\right): \operatorname{supp}(\mathbf{f}) \subset S\right\}
$$


For simplicity, we write $L^{2}(S)=L^{2}\left(S, \mathbb{C}^{1}\right)$. As pointed out in [23], Gabor analysis on $L^{2}\left(S, \mathbb{C}^{L}\right)$ with $S$ being $a \mathbb{Z}$-periodic might be suitable to treat periodic and intermittent vector-valued signals.

$\mathrm{Li}$ and Jia in [30] introduced and characterized scalar-valued weak Gabor bi-frames (WGBFs) under the setting of subspaces of $L^{2}(\mathbb{R})$. Tian and Li in [33] characterized Gabor bi-frames on time domain in the context of vector-valued subspaces. This paper addresses vector-valued subspace weak Gabor bi-frames under the following general setup:

General setup:

(i) $L \in \mathbb{N}, a, b>0$;

(ii) $S$ is an $a \mathbb{Z}$-periodic subset of $\mathbb{R}$.

We introduce the WGBF under the setting of vector-valued subspces, characterize WGBFs on the time domain, and also provide some examples of WGBFs. Theorem 3.1 in [30] dealt with WGBFs in scalar-valued subspaces of $L^{2}(\mathbb{R})$. Our result is a nontrivial generalization of [30], Theorem 3.1. This is because the inner product has a more complicated geometry in $L^{2}\left(\mathbb{R}, \mathbb{C}^{L}\right)$ than in $L^{2}(\mathbb{R})$.

Section 2 states the main result and some related remarks. Section 3 focuses on some lemmas, which is an auxiliary one. Section 4 is devoted to proving the main result. Some examples are provided simultaneously. Finally, we give our conclusions.

\section{Results and discussion}

Definition 2.1 Let $L, a, b$ and $S$ be as in the general setup, and $\mathbf{g}, \mathbf{h} \in L^{2}\left(S, \mathbb{C}^{L}\right)$. We say that

$$
(\mathcal{G}(\mathbf{g}, a, b), \mathcal{G}(\mathbf{h}, a, b))
$$

is a weak Gabor bi-frame $(W G B F)$ for $L^{2}\left(S, \mathbb{C}^{L}\right)$ if there exists a dense subset $\mathcal{M}$ of $L^{2}\left(S, \mathbb{C}^{L}\right)$ such that

$$
\langle\mathbf{f}, \tilde{\mathbf{f}}\rangle=\sum_{m, n \in \mathbb{Z}}\left\langle\mathbf{f}, E_{m b} T_{n a} \mathbf{g}\right\rangle\left\langle E_{m b} T_{n a} \mathbf{h}, \tilde{\mathbf{f}}\right\rangle \quad \text { for } \mathbf{f}, \tilde{\mathbf{f}} \in \mathcal{M}
$$

where the series is absolutely convergent. To be specific, in this case, we say that (4) is a weak Gabor bi-frame (WGBF) for $L^{2}\left(S, \mathbb{C}^{L}\right)$ related to $\mathcal{M}$.

Remark 2.1 Definition 2.1 reduces to [30], Definition 1.1, if $L=1$, where the authors dealt with the WGBFs for $L^{2}(S)$. In Definition 2.1, if $\mathcal{G}(\mathbf{g}, a, b)$ and $\mathcal{G}(\mathbf{h}, a, b)$ are Bessel sequences in $L^{2}\left(S, \mathbb{C}^{L}\right)$, then $(4)$ is a Gabor bi-frame $(G B F)$ for $L^{2}\left(S, \mathbb{C}^{L}\right)$ by a standard argument. So WGBF generalizes GBF. In applications, sometimes we only need to consider a certain class of signals (say the signals in $\mathcal{M}$ ) which is dense in $L^{2}\left(S, \mathbb{C}^{L}\right)$. Mathematically, it is roughly enough that (5) holds, no matter whether $\mathcal{G}(\mathbf{g}, a, b)$ and $\mathcal{G}(\mathbf{h}, a, b)$ are Bessel sequences. In this case, we can think

$$
\mathbf{f}=\sum_{m, n \in \mathbb{Z}}\left\langle\mathbf{f}, E_{m b} T_{n a} \mathbf{g}\right\rangle E_{m b} T_{n a} \mathbf{h}=\sum_{m, n \in \mathbb{Z}}\left\langle\mathbf{f}, E_{m b} T_{n a} \mathbf{h}\right\rangle E_{m b} T_{n a} \mathbf{g} \quad \text { for } \mathbf{f} \in \mathcal{M} .
$$

Remark 2.2 If $\mathcal{M}$ is a linear subspace of $L^{2}\left(S, \mathbb{C}^{L}\right)$, Eq. (5) is equivalent to

$$
\|\mathbf{f}\|^{2}=\sum_{m, n \in \mathbb{Z}}\left\langle\mathbf{f}, E_{m b} T_{n a} \mathbf{g}\right\rangle\left\langle E_{m b} T_{n a} \mathbf{h}, \mathbf{f}\right\rangle \quad \text { for } \mathbf{f} \in \mathcal{M}
$$


with the right-hand side series converging absolutely by the polarization identity of inner products.

Define the set $L_{c}^{\infty}\left(S, \mathbb{C}^{L}\right)$ as

$$
\left\{\mathbf{f}=\left(f_{1}, f_{2}, \ldots, f_{L}\right): f_{l} \in L^{\infty}(\mathbb{R}),\right.
$$

$f_{l}$ is compactly supported, and $\operatorname{supp}\left(f_{l}\right) \subset S$ for each $\left.1 \leq l \leq L\right\}$.

Obviously, $L_{c}^{\infty}\left(S, \mathbb{C}^{L}\right)$ is a dense subspace of $L^{2}\left(S, \mathbb{C}^{L}\right)$. The following theorem is our main result, it characterizes WGBFs for $L^{2}\left(S, \mathbb{C}^{L}\right)$ related to $L_{c}^{\infty}\left(S, \mathbb{C}^{L}\right)$ on the time domain.

Theorem 2.1 Let $L \in \mathbb{N}, a, b>0$, and $S$ be an aZZ-periodic subset of $\mathbb{R}$. Suppose $\mathbf{g}, \mathbf{h} \in$ $L^{2}\left(S, \mathbb{C}^{L}\right)$. Then $(\mathcal{G}(\mathbf{g}, a, b), \mathcal{G}(\mathbf{h}, a, b))$ is a WGBF for $L^{2}\left(S, \mathbb{C}^{L}\right)$ related to $L_{c}^{\infty}\left(S, \mathbb{C}^{L}\right)$ if and only if

$$
\sum_{n \in \mathbb{Z}} \overline{g_{l}\left(t-n a-\frac{k}{b}\right)} h_{j}(t-n a)=b \delta_{k, 0} \delta_{l, j} \chi_{S}(t)
$$

for $k \in \mathbb{Z}, 1 \leq l, j \leq L$ and a.e. $t \in(0, a)$.

Remark 2.3 Theorem 2.1 generalizes [30], Theorem 3.1, which dealt with the scalar case. It is a nontrivial generalization due to the complicated geometry of the inner product in $L^{2}\left(S, \mathbb{C}^{L}\right)$. This can be seen from its proof in Sect. 4 .

\section{Some lemmas}

This section is devoted to some lemmas which are used in the following section. We denote by $L^{\infty}\left(\mathbb{R}, \mathbb{C}^{L}\right)$ the Hilbert space

$$
L^{\infty}\left(\mathbb{R}, \mathbb{C}^{L}\right)=\left\{\mathbf{f}=\left(f_{1}, f_{2}, \ldots, f_{L}\right): f_{l} \in L^{\infty}(\mathbb{R}) \text { for each } 1 \leq l \leq L\right\}
$$

with the norm

$$
\|\mathbf{f}\|_{\infty}=\max _{1 \leq l \leq L}\left\|f_{l}\right\|_{\infty}
$$

Lemma 3.1 Let $L, b$ be as in the general setup, and $\mathbf{g}, \mathbf{h} \in L^{2}\left(\mathbb{R}, \mathbb{C}^{L}\right)$. Suppose $\mathbf{f}, \tilde{\mathbf{f}} \in$ $L^{2}\left(\mathbb{R}, \mathbb{C}^{L}\right)$ such that $f_{l}, \tilde{f}_{l}$ satisfying $\sum_{k \in \mathbb{Z}}\left|f_{l}\left(\cdot-\frac{k}{b}\right)\right|^{2}, \sum_{k \in \mathbb{Z}}\left|\tilde{f}_{l}\left(\cdot-\frac{k}{b}\right)\right|^{2} \in L^{\infty}\left(\left[0, \frac{1}{b}\right)\right)$ for each $1 \leq l \leq L$. Then

$$
\begin{aligned}
& \sum_{m \in \mathbb{Z}}\left\langle\mathbf{f}, E_{m b} \mathbf{g}\right\rangle\left\langle E_{m b} \mathbf{h}, \tilde{\mathbf{f}}\right\rangle \\
& \quad=\frac{1}{b} \sum_{1 \leq l, j \leq L} \int_{\mathbb{R}} \overline{\tilde{f}_{j}(t)} h_{j}(t) \sum_{k \in \mathbb{Z}} \overline{g_{l}\left(t-\frac{k}{b}\right)} f_{l}\left(t-\frac{k}{b}\right) d t
\end{aligned}
$$

with the left-hand side series converging absolutely. 
Proof By the assumptions of $\mathbf{f}, \tilde{\mathbf{f}}$, we see that $\sum_{l=1}^{L} \sum_{k \in \mathbb{Z}}\left|f_{l}\left(\cdot-\frac{k}{b}\right)\right|^{2} \leq M, \sum_{l=1}^{L} \sum_{k \in \mathbb{Z}} \tilde{f}_{l}(\cdot-$ $\left.\frac{k}{b}\right)\left.\right|^{2} \leq M$ for some $0<M<\infty$ and each $1 \leq l \leq L$. Then

$$
\begin{aligned}
& \sum_{l=1}^{L} \sum_{k \in \mathbb{Z}}\left|f_{l}\left(t-\frac{k}{b}\right) g_{l}\left(t-\frac{k}{b}\right)\right| \\
& \leq \sum_{l=1}^{L}\left(\sum_{k \in \mathbb{Z}}\left|f_{l}\left(t-\frac{k}{b}\right)\right|^{2}\right)^{\frac{1}{2}}\left(\sum_{k \in \mathbb{Z}}\left|g_{l}\left(t-\frac{k}{b}\right)\right|^{2}\right)^{\frac{1}{2}} \\
& \leq\left(\sum_{l=1}^{L} \sum_{k \in \mathbb{Z}}\left|f_{l}\left(t-\frac{k}{b}\right)\right|^{2}\right)^{\frac{1}{2}}\left(\sum_{l=1}^{L} \sum_{k \in \mathbb{Z}}\left|g_{l}\left(t-\frac{k}{b}\right)\right|^{2}\right)^{\frac{1}{2}} \\
& \leq \sqrt{M}\left(\sum_{l=1}^{L} \sum_{k \in \mathbb{Z}}\left|g_{l}\left(t-\frac{k}{b}\right)\right|^{2}\right)^{\frac{1}{2}} .
\end{aligned}
$$

And thus

$$
\begin{aligned}
& \int_{0}^{\frac{1}{b}}\left(\sum_{l=1}^{L} \sum_{k \in \mathbb{Z}}\left|f_{l}\left(t-\frac{k}{b}\right) g_{l}\left(t-\frac{k}{b}\right)\right|\right)^{2} d t \\
& \quad \leq M \int_{0}^{\frac{1}{b}} \sum_{l=1}^{L} \sum_{k \in \mathbb{Z}}\left|g_{l}\left(t-\frac{k}{b}\right)\right|^{2} d t \\
& \quad \leq M\|\mathbf{g}\|^{2} \\
& \quad<\infty .
\end{aligned}
$$

Similarly,

$$
\int_{0}^{\frac{1}{b}}\left(\sum_{l=1}^{L} \sum_{k \in \mathbb{Z}}\left|\tilde{f}_{l}\left(t-\frac{k}{b}\right) h_{l}\left(t-\frac{k}{b}\right)\right|\right)^{2} d t \leq M\|\mathbf{h}\|^{2}<\infty .
$$

By a simple calculation, we have

$$
\begin{aligned}
& \left\langle\mathbf{f}, E_{m b} \mathbf{g}\right\rangle=\int_{0}^{\frac{1}{b}}\left(\sum_{l=1}^{L} \sum_{k \in \mathbb{Z}} f_{l}\left(t-\frac{k}{b}\right) \overline{g_{l}\left(t-\frac{k}{b}\right)}\right) e^{-2 \pi i m b t} d t, \\
& \left\langle E_{m b} \mathbf{h}, \tilde{\mathbf{f}}\right\rangle=\int_{0}^{\frac{1}{b}}\left(\sum_{l=1}^{L} \sum_{k \in \mathbb{Z}} \overline{\tilde{f}_{l}\left(t-\frac{k}{b}\right)} h_{l}\left(t-\frac{k}{b}\right)\right) e^{2 \pi i m b t} d t,
\end{aligned}
$$

for $m \in \mathbb{Z}$. Again using (8) and (9), we see that the series $\sum_{m \in \mathbb{Z}}\left\langle\mathbf{f}, E_{m b} \mathbf{g}\right\rangle\left\langle E_{m b} \mathbf{h}, \tilde{\mathbf{f}}\right\rangle$ converges absolutely, and thus

$$
\begin{aligned}
& \sum_{m \in \mathbb{Z}}\left\langle\mathbf{f}, E_{m b} \mathbf{g}\right\rangle\left\langle E_{m b} \mathbf{h}, \tilde{\mathbf{f}}\right\rangle \\
& \quad=\frac{1}{b} \int_{0}^{\frac{1}{b}}\left(\sum_{l=1}^{L} \sum_{k \in \mathbb{Z}} f_{l}\left(t-\frac{k}{b}\right) \overline{g_{l}\left(t-\frac{k}{b}\right)}\right)\left(\sum_{j=1}^{L} \sum_{k \in \mathbb{Z}} \overline{\tilde{f}_{j}\left(t-\frac{k}{b}\right)} h_{j}\left(t-\frac{k}{b}\right)\right) d t,
\end{aligned}
$$


because of $\left\{\sqrt{b} e^{2 \pi i m b t}: m \in \mathbb{Z}\right\}$ being an orthonormal basis for $L^{2}\left(\left[0, \frac{1}{b}\right)\right)$. Write

$$
J(t)=\sum_{l=1}^{L} \sum_{k \in \mathbb{Z}} f_{l}\left(t-\frac{k}{b}\right) \overline{g_{l}\left(t-\frac{k}{b}\right)}
$$

Then (10) is equivalent to

$$
\sum_{m \in \mathbb{Z}}\left\langle\mathbf{f}, E_{m b} \mathbf{g}\right\rangle\left\langle E_{m b} \mathbf{h}, \tilde{\mathbf{f}}\right\rangle=\frac{1}{b} \int_{0}^{\frac{1}{b}} \sum_{j=1}^{L} \sum_{k \in \mathbb{Z}} \overline{\tilde{f}_{j}\left(t-\frac{k}{b}\right)} h_{j}\left(t-\frac{k}{b}\right) J(t) d t .
$$

Observing that

$$
\begin{aligned}
& \sum_{j=1}^{L} \sum_{k \in \mathbb{Z}}\left|\overline{\tilde{f}_{j}\left(t-\frac{k}{b}\right)} h_{j}\left(t-\frac{k}{b}\right) J(t)\right| \\
& \quad \leq\left(\sum_{j=1}^{L} \sum_{k \in \mathbb{Z}}\left|\tilde{f}_{j}\left(t-\frac{k}{b}\right) h_{j}\left(t-\frac{k}{b}\right)\right|\right)\left(\sum_{l=1}^{L} \sum_{k \in \mathbb{Z}}\left|f_{l}\left(t-\frac{k}{b}\right) g_{l}\left(t-\frac{k}{b}\right)\right|\right) \in L^{1}\left(\left[0, \frac{1}{b}\right)\right)
\end{aligned}
$$

by (8) and (9), we have

$$
\begin{aligned}
& \sum_{m \in \mathbb{Z}}\left\langle\mathbf{f}, E_{m b} \mathbf{g}\right\rangle\left\langle E_{m b} \mathbf{h}, \tilde{\mathbf{f}}\right\rangle \\
& =\frac{1}{b} \sum_{j=1}^{L} \sum_{k \in \mathbb{Z}} \int_{0}^{\frac{1}{b}} \overline{\tilde{f}_{j}\left(t-\frac{k}{b}\right)} h_{j}\left(t-\frac{k}{b}\right) J(t) d t \\
& =\frac{1}{b} \sum_{j=1}^{L} \int_{\mathbb{R}} \overline{\tilde{f}_{j}(t)} h_{j}(t) J(t) d t
\end{aligned}
$$

by the Lebesgue dominated convergence theorem. It follows that

$$
\begin{aligned}
& \sum_{m \in \mathbb{Z}}\left\langle\mathbf{f}, E_{m b} \mathbf{g}\right\rangle\left\langle E_{m b} \mathbf{h}, \tilde{\mathbf{f}}\right\rangle \\
& \quad=\frac{1}{b} \sum_{1 \leq l, j \leq L} \int_{\mathbb{R}} \overline{\tilde{f}_{j}(t)} h_{j}(t) \sum_{k \in \mathbb{Z}} \overline{g_{l}\left(t-\frac{k}{b}\right)} f_{l}\left(t-\frac{k}{b}\right) d t .
\end{aligned}
$$

Lemma 3.2 Let $L$, $a$ and $b$ be as in the general setup, and $\mathbf{g}, \mathbf{h} \in L^{2}\left(\mathbb{R}, \mathbb{C}^{L}\right)$. Then, for $\mathbf{f}, \tilde{\mathbf{f}} \in$ $L_{c}^{\infty}\left(\mathbb{R}, \mathbb{C}^{L}\right)$, we have

$$
\begin{aligned}
& \sum_{m, n \in \mathbb{Z}}\left\langle\mathbf{f}, E_{m b} T_{n a} \mathbf{g}\right\rangle\left\langle E_{m b} T_{n a} \mathbf{h}, \tilde{\mathbf{f}}\right\rangle \\
& \quad=\frac{1}{b} \sum_{1 \leq l, j \leq L} \int_{\mathbb{R}} \sum_{k \in \mathbb{Z}}\left(\sum_{n \in \mathbb{Z}} \overline{g_{l}\left(t-n a-\frac{k}{b}\right)} h_{j}(t-n a)\right) f_{l}\left(t-\frac{k}{b}\right) \overline{\tilde{f}_{j}(t)} d t
\end{aligned}
$$

with the left-hand side series converging absolutely. 
Proof Fix f $\tilde{\mathbf{f}} \in L_{c}^{\infty}\left(\mathbb{R}, \mathbb{C}^{L}\right)$. It is easy to check $\sum_{l=1}^{L} \sum_{k \in \mathbb{Z}}\left|f_{l}\left(\cdot-\frac{k}{b}\right)\right|^{2}, \sum_{l=1}^{L} \sum_{k \in \mathbb{Z}}\left|\tilde{f}_{l}\left(\cdot-\frac{k}{b}\right)\right|^{2} \in$ $L^{\infty}\left(\left[0, \frac{1}{b}\right)\right)$. For an arbitrary $n \in \mathbb{Z}$, replace $\mathbf{g}$ and $\mathbf{h}$ in Lemma 3.1 by $T_{n a} \mathbf{g}$ and $T_{n a} \mathbf{h}$, respectively. Then

$$
\begin{aligned}
& \sum_{m \in \mathbb{Z}}\left\langle\mathbf{f}, E_{m b} T_{n a} \mathbf{g}\right\rangle\left\langle E_{m b} T_{n a} \mathbf{h}, \tilde{\mathbf{f}}\right\rangle \\
& \quad=\frac{1}{b} \sum_{1 \leq l, j \leq L} \int_{\mathbb{R}} \sum_{k \in \mathbb{Z}} \overline{g_{l}\left(t-n a-\frac{k}{b}\right)} h_{j}(t-n a) f_{l}\left(t-\frac{k}{b}\right) \overline{\tilde{f}_{j}(t)} d t .
\end{aligned}
$$

Noting that $\mathcal{G}(\mathbf{f}, a, b)$ is a Bessel sequence by [24], Proposition 6.2.2, and

$$
\begin{aligned}
& \left|\left\langle\mathbf{f}, E_{m b} T_{n a} \mathbf{g}\right\rangle\right|=\left|\left\langle E_{-m b} T_{-n a} \mathbf{f}, \mathbf{g}\right\rangle\right|, \\
& \left|\left\langle\tilde{\mathbf{f}}, E_{m b} T_{n a} \mathbf{h}\right\rangle\right|=\left|\left\langle E_{-m b} T_{-n a} \tilde{\mathbf{f}}, \mathbf{h}\right\rangle\right|,
\end{aligned}
$$

we have $\left\{\left\langle\mathbf{f}, E_{m b} T_{n a} \mathbf{g}\right\rangle\right\}_{m, n \in \mathbb{Z}},\left\{\left\langle\tilde{\mathbf{f}}, E_{m b} T_{n a} \mathbf{h}\right\rangle\right\}_{m, n \in \mathbb{Z}} \in l^{2}\left(\mathbb{Z}^{2}\right)$, and thus the left-hand side series of Eq. (11) is absolutely convergent. Hence,

$$
\begin{aligned}
& \sum_{m, n \in \mathbb{Z}}\left\langle\mathbf{f}, E_{m b} T_{n a} \mathbf{g}\right\rangle\left\langle E_{m b} T_{n a} \mathbf{h}, \tilde{\mathbf{f}}\right\rangle \\
& \quad=\sum_{n \in \mathbb{Z}} \sum_{m \in \mathbb{Z}}\left\langle\mathbf{f}, E_{m b} T_{n a} \mathbf{g}\right\rangle\left\langle E_{m b} T_{n a} \mathbf{h}, \tilde{\mathbf{f}}\right\rangle \\
& \quad=\frac{1}{b} \sum_{1 \leq l, j \leq L} \sum_{n \in \mathbb{Z}} \int_{\mathbb{R}} \sum_{k \in \mathbb{Z}} \overline{g_{l}\left(t-n a-\frac{k}{b}\right)} h_{j}(t-n a) f_{l}\left(t-\frac{k}{b}\right) \overline{\tilde{f}_{j}(t)} d t
\end{aligned}
$$

by (12). Assume that $\operatorname{supp}\left(f_{l}\right), \operatorname{supp}\left(\tilde{f}_{l}\right) \subset[-N a, N a]$ for some $N \in \mathbb{N}$ and each $1 \leq l \leq L$. Then there exists $P \in \mathbb{N}$ such that $f_{l}\left(t-\frac{k}{b}\right) \tilde{\tilde{f}}_{j}(t)=0$ for $|k| \geq P$ and $1 \leq l, j \leq L$. And thus

$$
\begin{aligned}
& \int_{\mathbb{R}} \sum_{n \in \mathbb{Z}} \sum_{k \in \mathbb{Z}}\left|g_{l}\left(t-n a-\frac{k}{b}\right) h_{j}(t-n a) f_{l}\left(t-\frac{k}{b}\right) \tilde{f}_{j}(t)\right| d t \\
& \quad=\int_{-N a}^{N a} \sum_{n \in \mathbb{Z}} \sum_{|k| \leq P}\left|g_{l}\left(t-n a-\frac{k}{b}\right) h_{j}(t-n a) f_{l}\left(t-\frac{k}{b}\right) \tilde{f}_{j}(t)\right| d t \\
& \leq\|\mathbf{f}\|_{\infty}\|\tilde{\mathbf{f}}\|_{\infty} \sum_{|k| \leq P} \sum_{n \in \mathbb{Z}} \int_{-N a}^{N a}\left|g_{l}\left(t-n a-\frac{k}{b}\right) h_{j}(t-n a)\right| d t \\
& =\|\mathbf{f}\|_{\infty}\|\tilde{\mathbf{f}}\|_{\infty} \sum_{|k| \leq P} \sum_{n \in \mathbb{Z}} \sum_{j=-N}^{N-1} \int_{0}^{a}\left|g_{l}\left(t-(n-j) a-\frac{k}{b}\right) h_{j}(t-(n-j) a)\right| d t \\
& =\|\mathbf{f}\|_{\infty}\|\tilde{\mathbf{f}}\|_{\infty} \sum_{|k| \leq P} 2 N \sum_{n \in \mathbb{Z}} \int_{0}^{a}\left|g_{l}\left(t-n a-\frac{k}{b}\right) h_{j}(t-n a)\right| d t \\
& \quad=2 N\|\mathbf{f}\|_{\infty}\|\tilde{\mathbf{f}}\|_{\infty} \sum_{|k| \leq P} \int_{\mathbb{R}}\left|g_{l}\left(t-\frac{k}{b}\right) h_{j}(t)\right| d t \\
& <\infty
\end{aligned}
$$


for $1 \leq l, j \leq L$. Again using (13), we obtain

$$
\begin{aligned}
& \sum_{m, n \in \mathbb{Z}}\left\langle\mathbf{f}, E_{m b} T_{n a} \mathbf{g}\right\rangle\left\langle E_{m b} T_{n a} \mathbf{h}, \tilde{\mathbf{f}}\right\rangle \\
& \quad=\frac{1}{b} \sum_{1 \leq l, j \leq L} \int_{\mathbb{R}} \sum_{k \in \mathbb{Z}}\left(\sum_{n \in \mathbb{Z}} \overline{g_{l}\left(t-n a-\frac{k}{b}\right)} h_{j}(t-n a)\right) f_{l}\left(t-\frac{k}{b}\right) \overline{\tilde{f}_{j}(t)} d t .
\end{aligned}
$$

\section{Proof of Theorem 2.1 and some examples}

Proof of Theorem 2.1. Observe that $L_{c}^{\infty}\left(S, \mathbb{C}^{L}\right)$ is a linear dense subspace of $L^{2}\left(S, \mathbb{C}^{L}\right)$. It follows that (4) being a WGBF for $L^{2}\left(S, \mathbb{C}^{L}\right)$ related to $L_{c}^{\infty}\left(S, \mathbb{C}^{L}\right)$ is equivalent to

$$
\langle\mathbf{f}, \tilde{\mathbf{f}}\rangle=\frac{1}{b} \sum_{1 \leq l, j \leq L} \int_{\mathbb{R}} \sum_{k \in \mathbb{Z}}\left(\sum_{n \in \mathbb{Z}} \overline{g_{l}\left(t-n a-\frac{k}{b}\right)} h_{j}(t-n a)\right) f_{l}\left(t-\frac{k}{b}\right) \overline{\tilde{f}_{j}(t)} d t
$$

for $\mathbf{f}, \tilde{\mathbf{f}} \in L_{c}^{\infty}\left(S, \mathbb{C}^{L}\right)$ by Definition 2.1 and Lemma 3.2.

The proof of sufficiency is obvious. Next we will prove the necessity. Since

$$
\begin{aligned}
& \int_{0}^{a} \sum_{n \in \mathbb{Z}}\left|\overline{g_{l}\left(t-n a-\frac{k}{b}\right)} h_{j}(t-n a)\right| d t \\
& \leq \int_{0}^{a}\left(\sum_{n \in \mathbb{Z}}\left|g_{l}\left(t-n a-\frac{k}{b}\right)\right|^{2}\right)^{\frac{1}{2}}\left(\sum_{n \in \mathbb{Z}}\left|h_{j}(t-n a)\right|^{2}\right)^{\frac{1}{2}} d t \\
& \leq\left(\int_{0}^{a} \sum_{n \in \mathbb{Z}}\left|g_{l}\left(t-n a-\frac{k}{b}\right)\right|^{2} d t\right)^{\frac{1}{2}}\left(\int_{0}^{a} \sum_{n \in \mathbb{Z}}\left|h_{j}(t-n a)\right|^{2} d t\right)^{\frac{1}{2}} \\
& \quad=\left\|g_{l}\right\|_{L^{2}(\mathbb{R})}\left\|h_{j}\right\|_{L^{2}(\mathbb{R})}<\infty,
\end{aligned}
$$

almost all points in $(0, a)$ are Lebesgue points of

$$
\sum_{n \in \mathbb{Z}} \overline{g_{l}\left(t-n a-\frac{k}{b}\right)} h_{j}(t-n a) \quad \text { for } 1 \leq l, j \leq L, k \in \mathbb{Z}
$$

Fix $1 \leq l_{0}, j_{0} \leq L, k_{0} \in \mathbb{Z}$ and such a Lebesgue point $t_{0} \in(0, a)$. Take $0<\epsilon<\frac{1}{2 b}$ such that $B_{\epsilon}=\left(t_{0}-\epsilon, t_{0}+\epsilon\right) \subset(0, a)$, and define $\mathbf{f}, \tilde{\mathbf{f}}$ in (14) by

$$
\begin{aligned}
& \mathbf{f}=\left(0, \ldots, 0, f_{l_{0}}, 0, \ldots, 0\right), \quad f_{l_{0}}\left(t-\frac{k_{0}}{b}\right)=\frac{\chi_{B_{\epsilon}}(t) \chi_{S}(t)}{\sqrt{2 \epsilon}}, \\
& \tilde{\mathbf{f}}=\left(0, \ldots, 0, \tilde{f}_{j_{0}}, 0, \ldots, 0\right), \quad \tilde{f}_{j_{0}}(t)=\frac{\chi_{B_{\epsilon}}(t) \chi_{S}(t)}{\sqrt{2 \epsilon}} .
\end{aligned}
$$

Then

$$
\begin{aligned}
\int_{\mathbb{R}} & \frac{\chi_{B_{\epsilon}}(t) \chi_{S}(t)}{\sqrt{2 \epsilon}} \frac{\chi_{B_{\epsilon}}\left(t+\frac{k_{0}}{b}\right) \chi_{S}\left(t+\frac{k_{0}}{b}\right)}{\sqrt{2 \epsilon}} d t \\
= & \frac{1}{b} \int_{\mathbb{R}} \sum_{n \in \mathbb{Z}} \frac{g_{l_{0}}\left(t-n a-\frac{k_{0}}{b}\right)}{b} h_{l_{0}}(t-n a) \frac{\chi_{B_{\epsilon}}(t) \chi_{S}(t)}{2 \epsilon} d t
\end{aligned}
$$


when $l_{0}=j_{0}$, and

$$
0=\frac{1}{b} \int_{\mathbb{R}} \sum_{n \in \mathbb{Z}} \overline{g_{l_{0}}\left(t-n a-\frac{k_{0}}{b}\right)} h_{j_{0}}(t-n a) \frac{\chi_{B_{\epsilon}}(t) \chi_{S}(t)}{2 \epsilon} d t
$$

when $l_{0} \neq j_{0}$. From (15), we have

$$
\frac{1}{2 \epsilon} \int_{B_{\epsilon}} \delta_{k_{0}, 0} \chi_{S}(t) d t=\frac{1}{2 b \epsilon} \int_{B_{\epsilon}} \sum_{n \in \mathbb{Z}} \overline{g_{l_{0}}\left(t-n a-\frac{k_{0}}{b}\right)} h_{l_{0}}(t-n a) \chi_{S}(t) d t .
$$

This leads to

$$
\sum_{n \in \mathbb{Z}} \overline{g_{l_{0}}\left(t_{0}-n a-\frac{k_{0}}{b}\right)} h_{l_{0}}\left(t_{0}-n a\right) \chi_{S}\left(t_{0}\right)=b \delta_{k_{0}, 0} \chi_{S}\left(t_{0}\right)
$$

by letting $\epsilon \rightarrow 0$. Because of $h_{l_{0}} \in L^{2}(S)$, (17) is equivalent to

$$
\sum_{n \in \mathbb{Z}} \overline{g_{l_{0}}\left(t_{0}-n a-\frac{k_{0}}{b}\right)} h_{l_{0}}\left(t_{0}-n a\right)=b \delta_{k_{0}, 0} \chi_{S}\left(t_{0}\right)
$$

From (16), we have

$$
0=\frac{1}{2 b \epsilon} \int_{B_{\epsilon}} \sum_{n \in \mathbb{Z}} \overline{g_{l_{0}}\left(t-n a-\frac{k_{0}}{b}\right)} h_{j_{0}}(t-n a) \chi_{S}(t) d t .
$$

This leads to

$$
\sum_{n \in \mathbb{Z}} \overline{g_{l_{0}}\left(t_{0}-n a-\frac{k_{0}}{b}\right)} h_{j_{0}}\left(t_{0}-n a\right) \chi_{S}\left(t_{0}\right)=0
$$

by letting $\epsilon \rightarrow 0$. Because of $h_{j_{0}} \in L^{2}(S)$, (19) is equivalent to

$$
\sum_{n \in \mathbb{Z}} \overline{g_{l_{0}}\left(t_{0}-n a-\frac{k_{0}}{b}\right)} h_{j_{0}}\left(t_{0}-n a\right)=0 .
$$

Combining (18) with (20), we have

$$
\sum_{n \in \mathbb{Z}} \overline{g_{l_{0}}\left(t_{0}-n a-\frac{k_{0}}{b}\right)} h_{j_{0}}\left(t_{0}-n a\right)=b \delta_{k_{0}, 0} \delta_{l_{0}, j_{0}} \chi_{S}\left(t_{0}\right)
$$

Therefore, (6) holds by the arbitrariness of $j_{0}, l_{0}, k_{0}$ and $t_{0}$.

Example 4.1 Let $L, a, b$ and $S$ be as in the general setup, and $a b \leq \frac{1}{2}$. Suppose $\mathbf{g}, \mathbf{h} \in$ $L^{2}\left(S, \mathbb{C}^{L}\right)$, satisfy $\operatorname{supp}(\mathbf{g}), \operatorname{supp}(\mathbf{h}) \subset(-a, a)$ and

$$
\overline{g_{l}(t)} h_{j}(t)+\overline{g_{l}(t-a)} h_{j}(t-a)=b \delta_{l, j} \chi_{S}(t)
$$

for $1 \leq l, j \leq L$ and a.e. $t \in(0, a)$. Then (4) is a WGBF for $L^{2}\left(S, \mathbb{C}^{L}\right)$ related to $L_{c}^{\infty}\left(S, \mathbb{C}^{L}\right)$. In particular, if $\mathbf{g}, \mathbf{h} \in L^{\infty}\left(\mathbb{R}, \mathbb{C}^{L}\right)$ in addition, (4) is a GBF for $L^{2}\left(S, \mathbb{C}^{L}\right)$. 
Proof By a standard argument, we have

$$
\sum_{n \in \mathbb{Z}} \overline{g_{l}\left(t-n a-\frac{k}{b}\right)} h_{j}(t-n a)=\overline{g_{l}\left(t-a-\frac{k}{b}\right)} h_{j}(t-a)+\overline{g_{l}\left(t-\frac{k}{b}\right)} h_{j}(t)
$$

for $k \in \mathbb{Z}, 1 \leq l, j \leq L$ and a.e. $t \in(0, a)$. Since $a b \leq \frac{1}{2}$, we have $(-a, a) \cap\left((-a, a)+\frac{k}{b}\right)=\emptyset$ for $0 \neq k \in \mathbb{Z}$. And because of $\operatorname{supp}(\mathbf{g}) \subset(-a, a)$, we have

$$
g_{l}\left(t-a-\frac{k}{b}\right)=g_{l}\left(t-\frac{k}{b}\right)=0
$$

for $0 \neq k \in \mathbb{Z}, 1 \leq l \leq L$ and a.e. $t \in(0, a)$. Using (22), we obtain

$$
\sum_{n \in \mathbb{Z}} \overline{g_{l}\left(t-n a-\frac{k}{b}\right)} h_{j}(t-n a)=0
$$

for $0 \neq k \in \mathbb{Z}, 1 \leq l, j \leq L$ and a.e. $t \in(0, a)$. It follows that

$$
\sum_{n \in \mathbb{Z}} \overline{g_{l}\left(t-n a-\frac{k}{b}\right)} h_{j}(t-n a)=b \delta_{k, 0} \delta_{l, j} \chi_{S}(t)
$$

for $k \in \mathbb{Z}, 1 \leq l, j \leq L$ and a.e. $t \in(0, a)$ by (21). Therefore (4) is a WGBF for $L^{2}\left(S, \mathbb{C}^{L}\right)$ related to $L_{c}^{\infty}\left(S, \mathbb{C}^{L}\right)$ by Theorem 2.1. Furthermore, suppose $\mathbf{g}, \mathbf{h} \in L^{\infty}\left(\mathbb{R}, \mathbb{C}^{L}\right)$ in addition, then $\mathbf{g}, \mathbf{h} \in L_{c}^{\infty}\left(\mathbb{R}, \mathbb{C}^{L}\right)$. Therefore, $\mathcal{G}(\mathbf{g}, a, b)$ and $\mathcal{G}(\mathbf{h}, a, b)$ are Bessel sequences by [24], Proposition 6.2.2. Then, using $\mathbf{g}, \mathbf{h} \in L^{2}\left(S, \mathbb{C}^{L}\right)$, we see that they are Bessel sequences in $L^{2}\left(S, \mathbb{C}^{L}\right)$. So $(4)$ is a GBF for $L^{2}\left(S, \mathbb{C}^{L}\right)$ by Remark 2.1 .

Example 4.2 Let $L=2, a, b>0$ satisfying $a b \leq \frac{1}{2}, 0<\alpha, \beta<\frac{1}{2}, 0<c \leq a$ and $S=$ $\bigcup_{n \in \mathbb{Z}}[(0, c)+n a]$. Define $\mathbf{g}(t)=\left(g_{1}(t), g_{2}(t)\right), \mathbf{h}(t)=\left(h_{1}(t), h_{2}(t)\right)$ as follows:

$$
\begin{aligned}
& g_{1}(t)=\chi_{(0, c)}(t) t^{\alpha}+\left(\overline{c_{1}}+\overline{c_{2}}\right) \chi_{(-a, c-a)}(t)(t+a)^{-\beta}, \\
& g_{2}(t)=\left(\overline{c_{1}}-\overline{c_{2}}\right) \chi_{(0, c)}(t) t^{\alpha}+\left(1+\overline{c_{1}^{2}}-\overline{c_{2}^{2}}\right) \chi_{(-a, c-a)}(t)(t+a)^{-\beta}, \\
& h_{1}(t)=b\left(1+c_{1}^{2}-c_{2}^{2}\right) \chi_{(0, c)}(t) t^{-\alpha}-b\left(c_{1}-c_{2}\right) \chi_{(-a, c-a)}(t)(t+a)^{\beta}, \\
& h_{2}(t)=-b\left(c_{1}+c_{2}\right) \chi_{(0, c)}(t) t^{-\alpha}+b \chi_{(-a, c-a)}(t)(t+a)^{\beta},
\end{aligned}
$$

where $c_{1}$ and $c_{2}$ are two complex constants. Then (4) is a WGBF for $L^{2}\left(S, \mathbb{C}^{2}\right)$ related to $L_{c}^{\infty}\left(S, \mathbb{C}^{2}\right)$, but it is not a GBF for $L^{2}\left(S, \mathbb{C}^{2}\right)$.

Proof By a simple argument, $\mathbf{g}$, $\mathbf{h}$ satisfy the assumptions of Example 4.1. So (4) is a WGBF for $L^{2}\left(S, \mathbb{C}^{2}\right)$ related to $L_{c}^{\infty}\left(S, \mathbb{C}^{2}\right)$. Observe that at least one of $\sum_{n \in \mathbb{Z}}\left|g_{1}(\cdot-n a)\right|^{2}$, $\sum_{n \in \mathbb{Z}}\left|g_{2}(\cdot-n a)\right|^{2}, \sum_{n \in \mathbb{Z}}\left|h_{1}(\cdot-n a)\right|^{2}$ and $\sum_{n \in \mathbb{Z}}\left|h_{2}(\cdot-n a)\right|^{2} \notin L^{\infty}(\mathbb{R})$. Then at least one of $\mathcal{G}\left(g_{1}, a, b\right), \mathcal{G}\left(g_{2}, a, b\right), \mathcal{G}\left(h_{1}, a, b\right)$ and $\mathcal{G}\left(h_{2}, a, b\right)$ is not a Bessel sequence in $L^{2}(\mathbb{R})$ by [35], Proposition 11.3.4. It follows that at least one of $\mathcal{G}(\mathbf{g}, a, b)$ and $\mathcal{G}(\mathbf{h}, a, b)$ is not a Bessel sequence in $L^{2}\left(S, \mathbb{C}^{2}\right)$. This shows that (4) is not a GBF for $L^{2}\left(S, \mathbb{C}^{2}\right)$. 


\section{Conclusions}

The construction of bi-frames is a fundamental problem in frame theory. In recent years, the study of vector-valued frames and subspace frames has interested many mathematicians due to their wide applications. The concept of weak bi-frame generalizes that of biframe, and it has potentials in broadening applications of the frame theory in computation. We in this paper introduce the WGBF under the setting of vector-valued subspaces, characterize WGBFs on the time domain, and present some examples. Our result generalizes that of scalar-valued functions in the literature. Due to the more complicated geometry of inner products in vector-valued function spaces than in scalar-valued function spaces, our result is not a trivial generalization.

\section{Acknowledgements}

The authors would like to thank the reviewers for their suggestions which greatly improves the readability of this article.

\section{Funding}

This work was supported by the National Natural Science Foundation of China (Grant No. 11271037).

\section{Competing interests}

The authors declare that they have no competing interests.

\section{Authors' contributions}

All authors contributed equally to the writing of this paper. All authors read and approved the final manuscript.

\section{Publisher's Note}

Springer Nature remains neutral with regard to jurisdictional claims in published maps and institutional affiliations.

Received: 1 February 2018 Accepted: 13 June 2018 Published online: 22 June 2018

References

1. Duffin, R.J., Schaeffer, A.C.: A class of nonharmonic Fourier series. Trans. Am. Math. Soc. 72, 341-366 (1952)

2. Gabor, D.: Theory of communication. J. Inst. Electr. Eng. (London) 93, 429-457 (1946)

3. Arefijamaal, A.A., Akbar, E.: Signal processing by alternate dual Gabor frames. Appl. Comput. Harmon. Anal. 35, 535-540 (2013)

4. Casazza, P.G.: Modern tools for Weyl-Heisenberg frame theory. Adv. Imaging Electron Phys. 115, 1-127 (2001)

5. Daubechies, I., Grossmann, A., Meyer, Y.: Painless nonorthogonal expansions. J. Math. Phys. 27, 1271-1283 (1986)

6. Gröchenig, K., Stöckler, J.: Gabor frames and totally positive functions. Duke Math. J. 162, 1003-1031 (2013)

7. Han, D., Larson, D.: Frames, Bases and Group Representations. Mem. Amer. Math. Soc., vol. 147. Amer. Math. Soc., Providence (2000)

8. Balan, R.: Density and redundancy of the noncoherent Weyl-Heisenberg superframes. Contemp. Math. 247, 29-41 (1999)

9. Balan, R.: Multiplexing of signals using superframes. In: Aldroubi, A., Laine, A. (eds.) Wavelets and Applications in Signal and Image Processing VIII. SPIE Proceedings, vol. 4119, pp. 118-130 (2000)

10. Abreu, L.D.: Sampling and interpolation in Bargmann-Fock spaces of polyanalytic functions. Appl. Comput. Harmon. Anal. 29, 287-302 (2010)

11. Doerfler, M., Romero, J.L.: Frames adapted to a phase-space cover. Constr. Approx. 39, 445-484 (2014)

12. Abreu, L.D.: Superframes and polyanalytic wavelets. J. Fourier Anal. Appl. 23, 1-20 (2017)

13. Abreu, L.D.: Super-wavelets versus poly-Bergman spaces. Integral Equ. Oper. Theory 73, 177-193 (2012)

14. Bhatt, G., Johnson, B.D., Weber, E.: Orthogonal wavelet frames and vector-valued wavelet transforms. Appl. Comput. Harmon. Anal. 23, 215-234 (2007)

15. Abreu, L.D.: On the structure of Gabor and super Gabor spaces. Monatshefte Math. 161, 237-253 (2010)

16. Akinlar, M.A., Gabardo, J.-P.: Oblique duals associated with rational subspace Gabor frames. J. Integral Equ. Appl. 20, 283-309 (2008)

17. Führ, H.: Simultaneous estimates for vector-valued Gabor frames of Hermite functions. Adv. Comput. Math. 29, 357-373 (2008)

18. Gabardo, J.-P., Han, D.: Subspace Weyl-Heisenberg frames. J. Fourier Anal. Appl. 7, 419-433 (2001)

19. Gabardo, J.-P., Han, D.: Balian-Low phenomenon for subspace Gabor frames. J. Math. Phys. 45, 3362-3378 (2004)

20. Gabardo, J.-P., Han, D.: The uniqueness of the dual of Weyl-Heisenberg subspace frames. Appl. Comput. Harmon. Anal. 17, 226-240 (2004)

21. Gabardo, J.-P., Han, D., Li, Y.Z.: Lattice tiling and density conditions for subspace Gabor frames. J. Funct. Anal. 265, 1170-1189 (2013)

22. Gabardo, J.-P., Li, Y.-Z:: Density results for Gabor systems associated with periodic subsets of the real line. J. Approx. Theory 157, 172-192 (2009)

23. Gabardo, J.-P., Li, Y.-Z.: Rational time-frequency Gabor frames associated with periodic subsets of the real line. Int. J. Wavelets Multiresolut. Inf. Process. 12, Article ID 1450013 (2014) 
24. Gröchenig, K.: Foundations of Time-Frequency Analysis. Birkhäuser, Boston (2001)

25. Gröchenig, K., Lyubarskii, Y.: Gabor (super)frames with Hermite functions. Math. Ann. 345, 267-286 (2009)

26. Guo, X., Diao, Y., Dai, X.: Weyl-Heisenberg frame wavelets with basic support. Contemp. Math. 414, 3-12 (2006)

27. Han, D.: Classification of finite group-frames and super-frames. Can. Math. Bull. 50, 85-96 (2007)

28. Han, D.: The existence of tight Gabor duals for Gabor frames and subspace Gabor frames. J. Funct. Anal. 256, 129-148 (2009)

29. Li, Z.-Y., Han, D.: Constructing super Gabor frames: the rational time-frequency lattice case. Sci. China Math. 53, 3179-3186 (2010)

30. Li, Y.-Z., Jia, H.-F.: Weak Gabor bi-frames on periodic subsets of the real line. Int. J. Wavelets Multiresolut. Inf. Process. 13, Article ID 1550046 (2015)

31. Li, Y.-Z., Zhang, Y.: Vector-valued Gabor frames associated with periodic subsets of the real line. Appl. Math. Comput. $253,102-115(2015)$

32. Lian, Q.-F., Li, Y.-Z.: Gabor frame sets for subspaces. Adv. Comput. Math. 34, 391-411 (2011)

33. Tian, Y., Li, Y.-Z:: Subspace dual super wavelet and Gabor frames. Sci. China Math. 60, 2429-2446 (2017)

34. Zhang, Y., Li, Y.-Z.: Rational time-frequency multi-window subspace Gabor frames and their Gabor duals. Sci. China Math. 57, 145-160 (2014)

35. Christensen, O.: An Introduction to Frames and Riesz Bases. Birkhäuser, Boston (2016)

\section{Submit your manuscript to a SpringerOpen ${ }^{\circ}$ journal and benefit from:}

- Convenient online submission

- Rigorous peer review

- Open access: articles freely available online

- High visibility within the field

- Retaining the copyright to your article

Submit your next manuscript at $\boldsymbol{~ s p r i n g e r o p e n . c o m ~}$ 